\title{
A three-dimensional phase field model coupled with a lattice kinetics solver for modeling crystal growth in furnaces with accelerated crucible rotation and traveling magnetic field
}

\author{
Guang Lin, ${ }^{\mathrm{a}, *}$ Jie Bao, ${ }^{\mathrm{b}}$ Zhijie Xu, ${ }^{\mathrm{b}}$ \\ ${ }^{a}$ Department of Mathematics, School of Mechanical Engineering, Purdue \\ University, West Lafayette, IN 47907 USA \\ ${ }^{\mathrm{b}}$ Pacific Northwest National Laboratory, Richland, WA 99352 USA
}

\begin{abstract}
In this study, we present a new three-dimensional numerical model for crystal growth in a vertical solidification system. This model accounts for buoyancy, accelerated crucible rotation technique (ACRT), and traveling magnetic field (TMF) induced convective flow and their effect on crystal growth and the chemical component's transport process. The evolution of the crystal growth interface is simulated using the phase-field method. A semi-implicit lattice kinetics solver based on the Boltzmann equation is employed to model the unsteady incompressible flow. A one-way coupled concentration transport model is used to simulate the component fraction variation in both the liquid and solid phases, which can be used to check the quality of the crystal growth. Numerical results indicate that ACRT can slightly increase the quality of grown crystal, but the effect of TMF on quality of grown crystal depends on the temperature profile of the ampoule wall. Finally, excellent scalability of our developed parallel methods is demonstrated on the three-dimensional cases.
\end{abstract}

Key words: Phase field; Crystal growth; Accelerated crucible rotation technique (ACRT); Traveling magnetic field (TMF); Lattice Kinetics; Modeling

* Corresponding author. Email:guanglin@purdue.edu

Preprint submitted to Elsevier Science

16 July 2014 


\section{Nomenclature}

$\alpha \quad$ thermal diffusion coefficient $\left[m^{2} s^{-1}\right]$

$\alpha_{l} \quad$ thermal diffusion coefficient of liquid phase $\left[\mathrm{m}^{2} \mathrm{~s}^{-1}\right]$

$\alpha_{s} \quad$ thermal diffusion coefficient of solid phase $\left[m^{2} s^{-1}\right]$

$\beta \quad$ kinetic coefficient $\left[s m^{-1}\right]$

$\beta_{T} \quad$ coefficient of thermal expansion $\left[K^{-1}\right]$

$\Delta t \quad$ time step interval $[s]$

$\Delta x \quad$ mesh size $[m]$

$\epsilon \quad$ phase field model parameter

$\eta \quad$ derivative of pressure with respect to density $\left[\mathrm{m}^{2} \mathrm{~s}^{-2}\right]$

$\Gamma \quad$ interface between liquid and solid phases

$\lambda$ parameter that controls the strength of the coupling between the phase and diffusion fields

$\mu \quad$ liquid dynamic viscosity $\left[\mathrm{kg} \mathrm{m}^{-1} \mathrm{~s}^{-1}\right]$

$\mu_{m} \quad$ magnetic permeability $\left[\mathrm{H} \mathrm{m}^{-1}\right]$

$\nabla \quad$ differential operator in Cartesian coordinate system

$\nabla^{2} \quad$ second-order differential operator in Cartesian coordinate system

$\nabla_{r, z}^{2} \quad$ second-order differential operator in axisymmetric coordinate system

$\Omega \quad$ entire domain containing both liquid and solid phase

$\omega \quad$ electric current angular frequency $[\mathrm{Hz}]$

$\Omega_{g} \quad$ domain occupied by new grown solid phase

$\Omega_{l} \quad$ domain occupied by the liquid phase

$\Omega_{s} \quad$ domain occupied by the solid phase

$\phi_{n} \quad$ electric current phase shift between coils

$\psi \quad$ phase field

$\rho \quad$ density $\left[\mathrm{kg} \mathrm{m}^{-3}\right]$

$\rho_{l} \quad$ density of liquid $\left[\mathrm{kg} \mathrm{m}^{-3}\right]$

$\rho_{s} \quad$ density of solid $\left[\mathrm{kg} \mathrm{m}^{-3}\right]$

$\sigma \quad$ electrical conductivity $\left[S \mathrm{~m}^{-1}\right]$

$\tau \quad$ characteristic time of attachment of atoms at the interface $[s]$

$\tau_{s l, 1}, \tau_{s l, 2}$ tangential coordinates of curvilinear coordinate system moving with the interface $[m]$

$\Upsilon \quad$ linear relaxation parameter

$\varsigma \quad$ values at the beginning of the time step

$\varsigma+1$ values at the end of the time step

$\vartheta \quad$ surface tension $\left[\mathrm{kg} \mathrm{s}^{-2}\right]$

$\vec{e}_{z} \quad$ unit vector of $\mathrm{z}$ direction $[m]$

$\vec{e}_{i l} \quad$ particle velocity $\left[\mathrm{m} \mathrm{s}^{-1}\right]$

$\vec{F} \quad$ total external forces $\left[\mathrm{kg} \mathrm{m}^{-2} \mathrm{~s}-2\right]$

$\vec{F}_{L} \quad$ time-averaged Lorentz force per volume $\left[\mathrm{kg} \mathrm{m}^{-2} \mathrm{~s}^{-2}\right]$

$\vec{g} \quad$ gravity acceleration $\left[m s^{-2}\right]$

$\vec{n}_{s l, x} \quad \mathrm{x}$ component of normal direction of the solid-liquid interface $[\mathrm{m}]$

$\vec{n}_{s l, y} \quad$ y component of normal direction of the solid-liquid interface $[\mathrm{m}]$ 
$\vec{n}_{s l, z} \quad \mathrm{z}$ component of normal direction of the solid-liquid interface $[\mathrm{m}]$

$\vec{n}_{s l} \quad$ normal direction of the solid-liquid interface $[\mathrm{m}]$

$\vec{S}_{0} \quad$ total momentum source term $\left[\mathrm{kg} \mathrm{m}^{-2} \mathrm{~s}^{-1}\right]$

$\vec{S}_{0 b} \quad$ momentum source caused by buoyancy force $\left[\mathrm{kg} \mathrm{m}^{-2} \mathrm{~s}^{-1}\right]$

$\vec{S}_{0 L} \quad$ momentum source caused by Lorentz force $\left[\mathrm{kg} \mathrm{m}^{-2} \mathrm{~s}^{-1}\right]$

$\vec{u} \quad$ velocity vector $\left[\mathrm{ms}^{-1}\right]$

$\vec{u}_{p} \quad$ velocity of particles in Boltzmann equation $\left[\mathrm{m} \mathrm{s}^{-1}\right]$

$\vec{u}_{\text {wall }} \quad$ velocity of the ampoule wall $\left[\mathrm{ms}^{-1}\right]$

$\vec{x} \quad$ position vector in Cartesian coordinate system $[m]$

$\widetilde{F}_{i l} \quad$ external forces in lattice scheme $\left[\mathrm{kg} \mathrm{m}^{-3} \mathrm{~s}^{-1}\right]$

$A_{1} \quad$ in-phase components of magnetic potential $\left[V \mathrm{~s} \mathrm{~m}^{-1}\right]$

$a_{1} \quad$ phase field model parameter

$A_{2} \quad$ out-of-phase components of magnetic potential $\left[V \mathrm{~s} \mathrm{~m}^{-1}\right]$

$a_{2} \quad$ phase field model parameter

$a_{s} \quad$ anisotropy strength

$c$ non-dimensional chemical components' concentration

$\left.c\right|_{l}$ non-dimensional chemical components' concentration at solid-liquid interface in liquid side

$\left.c\right|_{s}$ non-dimensional chemical components' concentration at solid-liquid interface in solid side

$C_{p}^{l} \quad$ specific heat capacity of liquid $\left[\mathrm{J} \mathrm{kg}^{-1} \mathrm{~K}^{-1}\right]$

$C_{p}^{s} \quad$ specific heat capacity of solid $\left[J \mathrm{~kg}^{-1} \mathrm{~K}^{-1}\right]$

$c_{r} \quad$ reference chemical components' concentration

$c_{l k s} \quad$ lattice sound speed $\left[\mathrm{m} \mathrm{s}^{-1}\right]$

$c_{l k} \quad$ reference lattice speed $\left[\mathrm{m} \mathrm{s}^{-1}\right]$

$d_{0} \quad$ capillary length $[m]$

$D_{l} \quad$ chemical component diffusion coefficient in liquid phase $\left[m^{2} s^{-1}\right]$

$D_{s} \quad$ chemical component diffusion coefficient in solid phase $\left[m^{2} s^{-1}\right]$

$f \quad$ particle distribution function $\left[\mathrm{kg} \mathrm{m}^{-3}\right]$

$f_{i l}^{e q} \quad$ discretized equilibrium Maxwell distribution $\left[\mathrm{kg} \mathrm{m}^{-3}\right]$

$f_{i l, \rho} \quad$ discretized particle distribution function for density $\left[\mathrm{kg} \mathrm{m}^{-3}\right]$

$f_{i l, \vec{u}} \quad$ discretized particle distribution function for velocity $\left[\mathrm{kg} \mathrm{m}^{-3}\right]$

$f_{i l} \quad$ discretized particle distribution function $\left[\mathrm{kg} \mathrm{m}^{-3}\right]$

$H \quad$ height of ampoule $[m]$

$h \quad$ heat transfer coefficient $\left[W m^{-2} K\right]$

$J_{0} \quad$ electric current density $\left[A \mathrm{~m}^{-2}\right]$

$k \quad$ segregation coefficient

$K_{l} \quad$ thermal conductivity of liquid $\left[W m^{-1} K^{-1}\right]$

$K_{s} \quad$ thermal conductivity of solid $\left[W \mathrm{~m}^{-1} K^{-1}\right]$

$L \quad$ latent heat $\left[J \mathrm{~kg}^{-1}\right]$

$N \quad$ total number of lattice directions excluding the stationary 0 direction

$n$ norm coordinate of ampoule wall $[\mathrm{m}]$

$n_{s l} \quad$ norm coordinate of curvilinear coordinate system moving with the interface $[m]$ 
$R \quad$ radius of ampoule $[m]$

$r \quad \mathrm{r}$ direction in axisymmetric coordinate system, distance from the centerline of ampoule $[\mathrm{m}]$

$S_{\psi} \quad$ thermal source term caused by phase filed transition $\left[K s^{-1}\right]$

$T$ temperature $[K]$

$t \quad$ time $[s]$

$T_{0} \quad$ reference temperature $[K]$

$T_{c} \quad$ cold end temperature $[K]$

$T_{h} \quad$ hot end temperature $[K]$

$T_{i} \quad$ interface temperature $[K]$

$T_{m} \quad$ melting temperature $[K]$

$T_{w} \quad$ ampoule wall temperature $[K]$

$U$ magnitude of velocity $\left[\mathrm{m} \mathrm{s}^{-1}\right]$

$u \quad$ velocity component in $\mathrm{x}$ direction $\left[\mathrm{m} \mathrm{s}^{-1}\right]$

$U_{\text {pull }}$ ampoule pulling speed $\left[\mathrm{m} \mathrm{s}^{-1}\right]$

$v \quad$ velocity component in y direction $\left[\mathrm{m} \mathrm{s}^{-1}\right]$

$V_{i} \quad$ normal interfacial velocity $\left[\mathrm{m} \mathrm{s}^{-1}\right]$

$W \quad$ interface thickness $[m]$

$w \quad$ velocity component in $\mathrm{z}$ direction $\left[\mathrm{m} \mathrm{s}^{-1}\right]$

$W_{0} \quad$ phase field model parameter for interface thickness $[m]$

$x, y, z$ coordinates in Cartesian coordinate system $[m]$

$z_{i} \quad$ position of solid-liquid interface $[\mathrm{m}]$

$z_{i 0} \quad$ initial position of solid-liquid interface $[\mathrm{m}]$

\section{Introduction}

Due to the importance of crystals in a number of medical imaging applications and radiation detection [1-3], numerical simulations of crystal growth from the melt in vertical gradient furnaces $[4,5]$ have attracted significant attention.

Vertical growth techniques include both high- and ambient- pressure methods with recent advances in ambient-pressure methods at the forefront [3]. Ambient-pressure methods offer reduced experimental complexity and have been shown to produce large single-crystal volumes with properties as good or better than high-pressure methods. Thus, there has been a shift toward low-pressure methods using vertical gradient furnaces and sealed ampoule growth [3]. However, material uniformity, property homogeneity, and crystal defects remain difficult problems to solve for certain systems grown in this manner, such as cadmium zinc telluride (CZT). Modeling and simulation techniques are promoted as vehicles toward understanding the solidification process in complex systems and are thought to provide a more systematic method for determining optimal growth conditions and improved materials. 
Recent advances in computer models for growth processes in the vertical gradient furnace have been useful in understanding the general effects of furnace operating conditions on the growth of crystals [6]. As such, computer models have become a valuable tool in furnace design and the optimization of operating conditions [7-11]. At the same time, most existing models use a simplistic description of the crystal/melt interface and its dynamic. Furthermore, in these models, it is assumed that latent heat dissipates without disturbing the continuity of the heat fluxes at the interface. This approach fails to account for the effects of crystal anisotropy and solidification kinetics, which may be important in the simulations of crystal dendritic growth or lateral overgrowth $[12,13]$. Applying a traveling magnetic field (TMF) to the vertical-gradient furnace is a direct way to introduce a body force in the direction of gravity [14-16]. TMF can adjust the magnitude of the force by adjusting the strength of the electric current. As such, we believe TMF can be used to affect crystal quality in the vertical solidification system. The accelerated crucible rotation technique (ACRT) is applied to the vertical solidification system to improve mixing in the melt [17-21], which can increase the quality of crystal growth. Already, we have developed a phase-field-based model to simulate crystal growth in the vertical gradient furnace [22]. The model accounts for anisotropy in kinetic and interfacial free energy coefficients, as well as the effect of front curvature on crystal growth. The model was used to study the effects of ACRT and TMF on crystal growth in a prototypical vertical gradient furnace.

The phase-field method has become a standard tool to solve free-boundary problems and simulate interfacial pattern formation phenomena in solidification and other systems [23-25]. The phase-field method avoids explicit front tracking by replacing sharp interfaces with spatial smoothly diffused boundaries between bulk phases. Thus, the phase-field method is suitable for simulating time-dependent free boundary problems, especially when complex geometries are present. In a phase field model, an order parameter, phase field variable $\psi$ is introduced to smoothly vary from one value in the liquid region to another one in the solid region. This model is not only used to simulate solidification but also is able to track solid-solid phase transformations and is suitable for general microstructural evolution problems. Thus, solidification and subsequent microstructural coarsening during high-temperature furnace processing all can be addressed within the same simulation framework. To simulate chemical component segregation and solute balance at the solid-liquid interface, a concentration-field transport equation is one-way coupled with the phase-field equation. Similar to the phase field, the concentration field also smoothly varies from one value in the liquid region to another one in the solid region because of the segregation. This smooth transition is controlled by the phase field variable $\psi$. Hence, updating the concentration field avoids tracking the sharp solid-liquid interfaces as well. 
The primary difficulty for simulating the fluid field in the liquid region is the time-dependent growth interface between the liquid and solid phases. Hence, instead of using the traditional computational fluid dynamics (CFD) method based on solving Navier-Stokes equations, a semi-implicit lattice kinetics model [26] based on the Boltzmann equation method [27-30] is used for solving the fluid. One advantage of the lattice method is how it deals with complicated or moving solid boundaries. In addition, it is well known that the original lattice Boltzmann model (LBM) shows high efficiency in parallel computation, and the lattice kinetics method [31] is designed to eliminate the need to store and transmit the elements of the particle velocity distribution function, which has been thought to theoretically reduce the data transmitted between compute nodes for massively parallel computation [26,32]. The semiimplicit lattice kinetics method is modified from the original lattice kinetics method and increases the stability for large time steps [26]. In the following section, we present the equations that govern the melt convection, heat conduction, and solidification processes in the vertical gradient furnace. In Section 3 , we present the numerical model and steps for solving the governing equations. In Section 4, we discuss the results of numerical simulations for different operational conditions.

\section{Governing Equations}

Melt flow and crystal growth in a vertical gradient furnace with TMF can be described by a Boltzmann equation, energy conservation equations, and a time-averaged Lorentz force equation in a Cartesian coordinate system, as depicted in Figure 1:

$$
\begin{gathered}
\frac{\partial f}{\partial t}+\vec{u}_{p} \cdot \nabla f=Q(f, f), \quad \vec{x} \in \Omega_{l}, \\
\frac{\int \vec{u}_{p} f d \vec{u}_{p}}{\int f d \vec{u}_{p}}=\vec{u}, \quad \vec{x} \in \Omega_{l}, \\
\frac{\partial T}{\partial t}+\vec{u} \cdot \nabla T=\frac{K_{l}}{\rho_{l} C_{p}^{l}} \nabla^{2} T, \quad \vec{x} \in \Omega_{l}, \\
\frac{\partial T}{\partial t}=\frac{K_{s}}{\rho_{s} C_{p}^{s}} \nabla^{2} T, \quad \vec{x} \in \Omega_{s}, \\
\rho L V_{i}=K_{s} \partial_{n} T_{s}-K_{l} \partial_{n} T_{l}, \quad \vec{x} \in \Gamma, \\
T_{i}-T_{m}=-\frac{\vartheta}{\rho L} K_{l} T_{m}-\beta V_{i}, \quad \vec{x} \in \Gamma, \\
\vec{F}_{L}=-\frac{1}{2} \sigma \omega\left(A_{1} \nabla A_{2}-A_{2} \nabla A_{1}\right), \quad \vec{x} \in \Omega_{l} .
\end{gathered}
$$


The definition of each symbol is listed in the nomenclature. $\Omega_{l}$ is the domain occupied by the liquid phase (melt), $\Omega_{s}$ is the domain occupied by the solid phase (crystal), and $\Gamma=\Omega_{s} \cap \Omega_{l}$ is the interface between crystal and melt. Equation (1) is a Boltzmann equation, which governs the fluid dynamics in the liquid phase. $f$ is the particle distribution function (PDF) and represents the probability of finding particles at position $\vec{x}$ with velocity $\vec{u}_{p}$ at time $t . Q()$ is the Boltzmann collision operator [33-35], which includes the external source terms. Equation (2) shows the relation between mesoscopic-scale variables $\left(\vec{u}_{p}\right.$ and $f$ ) and the macroscopic variable $(\vec{u})$. More details and numerical methods for solving the Boltzmann equation will be introduced in the following sections. Equations (3-6) are energy equations for liquid and solid phases, as well as the phases' transitions. $\rho$ is the mass density. In this study, $\rho$ is assumed to be the same for both liquid and solid phases. Temperature $T$ is a continuous variable in the whole domain, so the interface temperature $T_{i}$ is the same for liquid and solid phases. $L$ is the latent heat, and $V_{i}$ is the interface velocity along interface norm direction. $\partial_{n}$ is the gradient along the interface norm direction. Equation (7) expresses the time-averaged Lorentz force per volume $\vec{F}_{L} \cdot A_{1}$ and $A_{2}$ are in-phase and out-of-phase components of magnetic potential [16,36], and the meaning of symbols in equations are listed among the nomenclature. The stationary amplitudes of in-phase and out-of-phase components of the potential are calculated by solving the Maxwell's equation of Ampere's law in an axisymmetric coordinate system, as shown in Figure 2, where $z$ is the center axis of the cylinder:

with right-hand sides:

$$
\begin{aligned}
& \nabla_{r, z}^{2} A_{1}-\frac{A_{1}}{r^{2}}=R H S_{1}, \\
& \nabla_{r, z}^{2} A_{2}-\frac{A_{2}}{r^{2}}=R H S_{2},
\end{aligned}
$$

$$
\begin{gathered}
\text { in coils : } R H S_{1}=-\mu_{m} J_{0} \cos \phi_{n}, R H S_{2}=-\mu_{m} J_{0} \sin \phi_{n}, \\
\text { elsewhere }: R H S_{1}=\mu_{m} \sigma \omega A_{2}, R H S_{2}=-\mu_{m} \sigma \omega A_{1},
\end{gathered}
$$

where $J_{0}$ is electric current density, $\mu_{m}$ is magnetic permeability, and $\phi_{n}$ is phase shift at each coil.

The boundary conditions for the flow and energy equations (Eqs. (1-6)) are:

At the top of the crucible wall: The no-slip, no-flow boundary conditions are employed (i.e., $\vec{u}=\vec{u}_{\text {wall }}$, where $\vec{u}_{\text {wall }}$ is the velocity of the ampoule wall), and the thermal boundary condition is:

$$
\frac{\partial T}{\partial n}=0
$$

At the vertical crucible wall: The no-slip boundary conditions are employed at the vertical walls between the surrounding crucible and the liquid $\left(\vec{u}=\vec{u}_{\text {wall }}\right)$. 
Therefore, the velocity of melt is 0 at the ampoule walls for a furnace without rotation $\left(\vec{u}_{\text {wall }}=0\left[\mathrm{~m} \mathrm{~s}^{-1}\right]\right)$. For the ACRT test case, an ACRT rotation cycle is applied on the ampoule, as shown in Figure 3. Hence, the velocity of the ampoule wall and solid-liquid interface should be calculated according to the rotation rate. The thermal boundary conditions for the vertical crucible walls are:

$$
-K_{l} \frac{\partial T}{\partial n}=h\left(T-T_{w}(z)\right)
$$

where $h$ is the heat transfer coefficient and $T_{w}(z)$ is the ambient temperature inside the furnace along the quartz ampoule walls and can be calculated by Equation(14) [37].

$$
T_{w}(z)=\frac{1}{2}\left\{T_{c}+T_{h}+\left(T_{h}-T_{c}\right) \tanh \left[\left(\frac{d T_{w}(0)}{d z}\right) \frac{\left(z-z_{i}\right)}{\left(T_{h}-T_{c}\right)}\right]\right\},
$$

where $T_{c}$ is the cold-end temperature, $T_{h}$ is the hot-end temperature, and $\frac{d T_{w}(0)}{d z}$ is the maximum axial derivative of the ampoule wall temperature profile. The variable $z_{i}$ is the approximate position of the melt-solid interface. Generally, there are two main types of setup for applying the temperature profile $T_{w}(z)$ on the ampoule wall. The first one consists of choosing a reference frame fixed with the imposed temperature distribution on a stationary wall [38]. In this frame, the ampoule and solidified material move axially at pulling speed $\left(-U_{\text {pull }}\right)$. This is called a fixed temperature profile setup, and $z_{i}$ is a fixed value. The second setup involves letting the frame move with the moving ampoule, so the melt-solid interface grows approximately at velocity $U_{\text {pull }}$. This is called a time-dependent temperature profile setup, and $z_{i}=z_{i 0}+U_{\text {pull }}$. The first setup incurs less computational cost to update the computation mesh, so it is favorable for the interface front-tracking method [39-41]. In this paper, we show the phase field model does not need to track and update the computation mesh when the interface is moving. Thus, the computational cost for our model is the same as these two wall temperature profile setups. Additionally, if the concentration of a certain component in the solid phase during crystal growth is calculated in the model, the temperature boundary condition must be the second setup (already described herein). For Maxwell's equations (Eqs. 8 and 9 ), the boundary conditions are a zero gradient of in-phase and out-of-phase potential at the system outer shell and centerline, as illustrated in Figure 2.

The uniformity of the chemical components' concentration in the solid phase is a criterion of the quality of the crystal, such as zinc in a CZT crystal. To model the transport of a chemical component, it is assumed that the component diffuses as a dilute species:

$$
\frac{1}{D_{l}} \frac{\partial c}{\partial t}+\frac{1}{D_{l}} \vec{u} \cdot \nabla c=\nabla^{2} c, \quad \vec{x} \in \Omega_{l}
$$




$$
\begin{aligned}
\frac{1}{D_{s}} \frac{\partial c}{\partial t} & =\nabla^{2} c, \quad \vec{x} \in \Omega_{s}, \\
\left(-\left.D_{l} \nabla c\right|_{l}+\left.D_{s} \nabla c\right|_{s}\right) \cdot \vec{n}_{s l} & =-\left.(k-1) c\right|_{l} \times V_{i}\left(\vec{n}_{s l} \cdot \overrightarrow{e_{z}}\right), \quad \vec{x} \in \Gamma,
\end{aligned}
$$

where $c$ is a non-dimensional zinc concentration scaled by a reference concentration $c_{r} . D_{l}$ and $D_{s}$ are diffusion coefficients in liquid and solid phases, respectively. $\vec{n}_{s l}$ is the normal direction of the solid-liquid interface, and $V_{i}$ is the interface moving velocity. At the solid-liquid interface, the zinc concentration in the melt $\left(\left.c\right|_{l}\right)$ is related to the concentration in the solid $\left(\left.c\right|_{s}\right)$ by the equilibrium segregation coefficient $k$ :

$$
\left.c\right|_{s}=\left.k c\right|_{l}
$$

All other boundaries are assumed to be impermeable to zinc. This paper focuses on zinc segregation during CZT crystal growth, but the model introduced herein can be used for other components in the growth of different semiconductor crystals with few modifications.

\section{$3 \quad$ Numerical Methods}

Equations(1-6) constitute a (highly non-linear) free boundary problem due to the presence of moving solid-liquid interface $\Gamma$. The phase field method [24] is used to reduce the free boundary problem to a system of coupled Boltzmann, energy, and phase field equations. In the phase field equation, the state variable $\psi$ takes a value of 1 in the solid phase (crystal) and -1 in the liquid phase (melt).

$$
\begin{gathered}
f_{i l}\left(\vec{x}+\vec{e}_{i l} \Delta t, t+\Delta t\right)-f_{i l}(\vec{x}, t)=-\frac{1}{\Upsilon}\left[f_{i l}(\vec{x}, t)-f_{i l}^{e q}(\vec{x}, t)\right]+\Delta t \widetilde{F}_{i l}, \quad \vec{x} \in \Omega_{l}, \\
\frac{\partial T}{\partial t}+u \frac{\partial T}{\partial x}+v \frac{\partial T}{\partial y}+w \frac{\partial T}{\partial z}=\alpha_{l} \nabla^{2} T+S_{\psi}, \quad \vec{x} \in \Omega_{l}, \\
\frac{\partial T}{\partial t}=\alpha_{s} \nabla^{2} T, \quad \vec{x} \in \Omega_{s},
\end{gathered}
$$




$$
\begin{aligned}
& \tau \frac{\partial \psi}{\partial t}=\left[\psi-\lambda\left(1-\psi^{2}\right) \frac{T-T_{m}}{T_{0}}\right]\left(1-\psi^{2}\right)+W^{2} \nabla^{2} \psi \\
& +2 W \frac{\partial \psi}{\partial x} \frac{\partial W}{\partial x}+2 W \frac{\partial \psi}{\partial y} \frac{\partial W}{\partial y}+2 W \frac{\partial \psi}{\partial z} \frac{\partial W}{\partial z} \\
& +\frac{\partial}{\partial x}\left\{\left[\left(\partial_{x} \psi\right)^{2}+\left(\partial_{y} \psi\right)^{2}+\left(\partial_{z} \psi\right)^{2}\right] W \frac{\partial W}{\partial\left(\partial_{x} \psi\right)}\right\} \\
& +\frac{\partial}{\partial y}\left\{\left[\left(\partial_{x} \psi\right)^{2}+\left(\partial_{y} \psi\right)^{2}+\left(\partial_{z} \psi\right)^{2}\right] W \frac{\partial W}{\partial\left(\partial_{y} \psi\right)}\right\} \\
& +\frac{\partial}{\partial z}\left\{\left[\left(\partial_{x} \psi\right)^{2}+\left(\partial_{y} \psi\right)^{2}+\left(\partial_{z} \psi\right)^{2}\right] W \frac{\partial W}{\partial\left(\partial_{z} \psi\right)}\right\}, \quad \vec{x} \in \Omega,
\end{aligned}
$$

and

$$
W\left(\vec{n}_{s l}\right)=W_{0} a_{s}\left(\vec{n}_{s l}\right)^{2}, \quad \vec{x} \in \Omega,
$$

where $\Omega=\Omega_{l} \cup \Omega_{s}$. Equation (19) is the discretized lattice Boltzmann equation with a simple Bhatnagar-Gross-Krook (BGK) collision operator. The particle distribution function $f$ becomes a finite set of displacement vectors connecting each lattice site to adjacent sites, and the subscript $i l$ represents the lattice vector's components. For a three-dimensional D3Q19 lattice as shown in Figure $4, i l$ is from 0 to 18 , and $\vec{e}_{i l}$ is the particle velocity. The variable $f_{i l}^{e q}$ is the equilibrium Maxwell distribution [42], and $\widetilde{F}_{i l}$ is the external forcing term in the lattice scheme that can be calculated by [43]:

$$
\widetilde{F}_{i l}=\left(1-\frac{1}{2 \Upsilon}\right) w_{i l}\left(\frac{\vec{e}_{i l}-\vec{u}}{c_{l k s}^{2}}+\frac{\vec{e}_{i l} \cdot \vec{u}}{c_{l k s}^{4}} \vec{e}_{i}\right) \cdot \vec{F},
$$

where $\vec{F}$ is the external force, $w_{i l}$ is the weight coefficient (for a D3Q19 system: $w_{0}=1 / 3, w_{1-6}=1 / 18$ and, $\left.w_{7-18}=1 / 36[44]\right)$, and $c_{l k s}^{2}=(\Delta x / \Delta t)^{2} / 3$ is the lattice sound speed [45]. In the energy equations (Eqs. (20 and 21)), $\alpha_{l}$ and $\alpha_{s}$ are the thermal diffusivity of liquid and solid phases, which equal to $\frac{K_{l}}{C_{p}^{l} \rho_{l}}$ and $\frac{K_{s}}{C_{p}^{s} \rho_{s}}$, respectively. The model is valid only in the symmetric conductivity limit, so $\alpha_{l}=\alpha_{s}=\alpha$, and $\rho_{l}=\rho_{s}=\rho[24,46]$. The heat source term $S_{\psi}$ is caused by the phase transition, and it varies in different kinds of wall temperature profile setups. For the first case, fixed wall temperature profile setup, $S_{\psi}=\frac{L}{2 C_{p}} \frac{\partial \psi}{\partial z} U_{\text {pull }}$. For the second setup, time-dependent temperature profile setup, $S_{\psi}=\frac{L}{2 C_{p}} \frac{\partial \psi}{\partial t}[24]$. The source term in the second temperature profile setup can be transformed to the curvilinear coordinate system $\left(\tau_{s l, 1}\right.$, $\left.\tau_{s l, 2}, n_{s l}\right)$, where $\tau_{s l, 1}$ and $\tau_{s l, 2}$ are the two orthogonal tangential coordinates of the solid-liquid interface, and $n_{s l}$ is the normal direction of the interface pointing into liquid, moving with the interface at an instantaneous velocity of $V_{i}$ as $S_{\psi}=\frac{L}{2 C_{p}} \frac{\partial \psi}{\partial n_{s l}} V_{i}$. For a very slow pulling speed, $U_{\text {pull }}$ is approximately equivalent to $V_{i}$. For small interface curvature, $\frac{\partial}{\partial z}$ is equivalent to $\frac{\partial}{\partial n_{s l}}$ in the vertical solidification system. Therefore, the thermal source terms in the two setups are equivalent for the crystal growth in furnaces. Our previous work demonstrates this through example test cases [22]. According to Karma's work [24], 
the phase field equation is defined as Eqs. $(22)$ and $(23)$, in which $W\left(\vec{n}_{s l}\right)$ is the interface thickness, and $\tau\left(\vec{n}_{s l}\right)=\tau_{o} a_{s}\left(\vec{n}_{s l}\right)^{2}$. For crystals with four-fold symmetry, $a_{s}\left(\vec{n}_{s l}\right)=(1-3 \epsilon)\left\{1+[4 \epsilon /(1-3 \epsilon)]\left(n_{s l, x}^{4}+n_{s l, y}^{4}+n_{s l, z}^{4}\right)\right\}$ is anisotropy strength, where $n_{s l, x}, n_{s l, y}$, and $n_{s l, z}$ are the $x, y$, and $z$ component of the unit norm vector $\vec{n}_{s l}$. The parameter $\epsilon$ usually is measured experimentally by examining the deviation of an equilibrium shape from a circle [24]. $\epsilon=0.05$ is used in our study. In the sharp interface limit, the phase field parameters $\lambda$ and $\tau_{0}$ are related to the parameters $d_{0}$ and $W_{0}$ via:

$$
\lambda=a_{1} \frac{W_{0}}{d_{0}}, \quad \tau_{0}=\frac{a_{2} W_{0}^{2} \lambda}{\alpha}
$$

where $a_{1}=0.8839, a_{2}=0.6267$ [24], and $d_{0}=T_{m} C_{p} \vartheta / L^{2}$. To sufficiently resolve the phase field function, the grid size should satisfy the condition $W_{0} \leq 2.5 \Delta x$ [24]. $W_{0}=2.5 \Delta x$ is used in the tests that are presented herein. The proposed phase field model has been validated in a benchmark test in our previous work, and it shows that the simulation results can match the analytical solution perfectly for $W_{0}<706 d_{0}$ [22]. The kinetic coefficient $\beta$ is related to $\beta\left(\vec{n}_{s l}\right)=\frac{a_{1} \tau\left(\vec{n}_{s l}\right)}{\lambda W\left(\vec{n}_{s l}\right)}\left[1-a_{2} \lambda \frac{W\left(\vec{n}_{s l}\right)^{2}}{\alpha \tau\left(\vec{n}_{s l}\right)}\right]$.

After coupling with the phase field equation, the transport of the concentration of zinc and its boundary condition at the solid-liquid interface can be transformed to this form:

$$
\frac{\partial c}{\partial t}=D_{l} \nabla^{2} c-\frac{1}{2|\nabla \psi|} \frac{\partial \psi}{\partial t}\left[D_{l} \nabla^{2} \psi-2 \frac{\partial \psi}{\partial t}\right], \quad \vec{x} \in \Omega .
$$

The second term on the right-hand side of Equation 26 provides a solute sink or source term due to phase change. In the phase field model, this is a diffusive source or sink localized in the vicinity of the interface $(\partial \psi / \partial t$ is negligibly small outside of the diffuse interface region). This term is needed to ensure that the interface conditions between two phases converge to the sharp interface equation (Eq. (17)) when $W \rightarrow 0$. In our previous work, the requirement that the phase field model must converge to the correct sharp interface limit is rigorously satisfied by Equation $(26)$ [47,48]. First, Equation (26) can be transformed to the $\left(\tau_{s l, 1}, \tau_{s l, 2}, n_{s l}\right)$ curvilinear coordinate system moving with the interface at an instantaneous velocity of $V_{i}$. Then, Equation(26) can be rewritten as:

$$
\frac{D_{l}}{V_{i}} \frac{\partial^{2} c}{\partial n_{s l}^{2}}+\left(\frac{D_{l} \kappa}{V_{i}}+1\right) \frac{\partial c}{\partial n_{s l}}+V_{i} \frac{\partial \psi}{\partial n_{s l}}+\frac{D_{l}}{2}\left(\frac{\partial^{2} \psi}{\partial n_{s l}^{2}}+\kappa \frac{\partial \psi}{\partial n_{s l}}\right)=0
$$

where $\kappa$ is the interface curvature. Integrating both sides of Equation (27) across the interface in the sharp interface limit in which the phase field $\psi$ becomes a step function and velocity normal to the interface becomes the 
sharp interface velocity $\left(V_{i}\right)$ gives:

$$
\left.\frac{D_{l}}{V_{i}} \frac{\partial c}{\partial n_{s l}}\right|_{0^{-}} ^{0^{+}}+\left.\left(\frac{D_{l} \kappa}{V_{i}}+1\right) c\right|_{0^{-}} ^{0^{+}}+\left.V_{i} \psi\right|_{0^{-}} ^{0^{+}}+\frac{D_{l}}{2}\left(\left.\frac{\partial \psi}{\partial n_{s l}}\right|_{0^{-}} ^{0^{+}}+\kappa \psi||_{0^{-}}^{0^{+}}\right)=0 .
$$

In the sharp interface limit, a step function can be used for the order parameter profile. The term $\left.\right|_{0^{-}} ^{0^{+}}$represents the difference of a variable at two sides of the solid-liquid interface, so

$$
\begin{aligned}
& \left.c\right|_{0^{-}} ^{0^{+}}=\left.c\right|_{s}-\left.c\right|_{l}, \\
& \left.\frac{\partial c}{\partial n_{s l}}\right|_{0^{-}} ^{0^{+}}=\left.\frac{\partial c}{\partial n_{s l}}\right|_{s}-\left.\frac{\partial c}{\partial n_{s l}}\right|_{l}, \\
& \left.\psi\right|_{0^{-}} ^{0^{+}}=2, \\
& \left.\frac{\partial \psi}{\partial n_{s l}}\right|_{0^{-}} ^{0^{+}}=0 .
\end{aligned}
$$

Integrating Equation (27) twice across the interface as $\psi$ approaches the step function and $c$ approaches the exact solution to the sharp interface model gives:

$$
\left.\frac{D_{l}}{V_{i}} c\right|_{0^{-}} ^{0^{+}}+\left.\frac{D_{l}}{2} \psi\right|_{0^{-}} ^{0^{+}}=0
$$

After substituting Equation (29) into Equation (30), Equation (30) leads directly to the results:

$$
\left.c\right|_{0^{-}} ^{0^{+}}=-V_{i}
$$

Substituting Equation (31) into Equation (28) recovers the boundary condition for the sharp interface limit,

$$
\left.D_{l} \frac{\partial c}{\partial n_{s l}}\right|_{0^{-}} ^{0^{+}}=-\left.c\right|_{0^{-}} ^{0^{+}} V_{i}
$$

Substituting Equation (29) into Equation (32) and transforming it back to a three-dimensional Cartesian coordinate system, Equation (32) becomes the same as Equation (17). The concentration equation (Eq. (26)) is applicable in the whole simulation domain, and the boundary condition at the solid-liquid interface $(x \in \Gamma)$, described in Equation (17), can be applied automatically instead of explicitly tracking the position of the interface.

A semi-implicit lattice method [26] is employed to solve the lattice Boltzmann equation (Eq. (19)) for the fluid field. In the semi-implicit lattice method, the pressure and velocity can be updated using the following equations:

$$
p^{\varsigma+1}=\frac{p^{\varsigma}+\eta\left(\sum_{i l=1}^{N} f_{i l, \rho}^{\vec{x}-\overrightarrow{i l}}\right)^{\varsigma+1}-\eta \frac{3}{2} w_{0} \frac{\rho \vec{u} \cdot \vec{u}}{c_{l k}^{2}}}{1+\frac{\eta}{c_{l k s}^{2}}\left(1-w_{0}\right)},
$$




$$
\vec{u}^{\varsigma+1}=\frac{(\rho \vec{u})^{\varsigma}+\left(\sum_{i l=1}^{N} \overrightarrow{e_{i l}} f_{i l, \vec{u}}^{\vec{x}-\overrightarrow{i l}}\right)^{\varsigma+1}+\overrightarrow{S_{0}}}{\rho+\rho \frac{6 \nu}{c_{l k}^{2} \Delta t}+S_{1}},
$$

where $\eta$ is the derivative of pressure with respect to density. The superscript $\varsigma$ indicates values at the beginning of the time step, and $\varsigma+1$ represents estimated values at the end of the time step. $N$ is the total number of lattice directions, excluding the stationary 0 direction. The term $\nu$ is the fluid kinetic viscosity, and $c_{l k}=\Delta x / \Delta t$ is the reference lattice speed [45]. The term $\overrightarrow{S_{0}}$ is the momentum source. The buoyancy force and Lorentz force can be applied in the term $\overrightarrow{S_{0}}$ :

$$
\begin{aligned}
& \vec{S}_{0}=\vec{S}_{0 b}+\vec{S}_{0 L}, \\
& \text { buoyancy force : } \vec{S}_{0 b}=\vec{g} \rho \Delta t \beta_{T}\left(T-T_{0}\right), \\
& \text { Lorentzforce : } \vec{S}_{0 L}=\Delta t \vec{F}_{L},
\end{aligned}
$$

where $T_{0}$ is a reference temperature, $\vec{g}$ is gravity, and $\beta_{T}$ is thermal expansion. The term $S_{1}$ is a mass source, which can be used to emulate Darcy resistance in a porous medium [26]. In Equations (33) and (34), $f_{i l, \rho}^{\vec{x}-e_{i l}}$ and $f_{i l, \vec{u}}^{\vec{x}-\overrightarrow{i l}}$ represent the particle probability distribution arriving from the neighboring lattice site in the $-\overrightarrow{e_{i l}}$ direction, and they can be calculated by:

$$
\begin{gathered}
f_{i l, \rho}=w_{i l}\left[\frac{p}{c_{l k s}^{2}}+\rho \frac{3 \overrightarrow{i_{i l}} \cdot \vec{u}}{c_{l k}^{2}}+\rho\left(\frac{9\left(\overrightarrow{e_{i l}} \cdot \vec{u}\right)^{2}}{2 c_{l k}^{4}}-\frac{3 \vec{u} \cdot \vec{u}}{2 c_{l k}^{2}}\right)\right] \\
f_{i l, \vec{u}}=w_{i l}\left[\frac{p}{c_{l k s}^{2}}+\frac{6 \nu \rho}{c_{l k}^{2} \Delta t} \frac{3 \overrightarrow{e_{i l}} \cdot \vec{u}}{c_{l k}^{2}}+\rho\left(\frac{9\left(\overrightarrow{e_{i l}} \cdot \vec{u}\right)^{2}}{2 c_{l k}^{4}}-\frac{3 \vec{u} \cdot \vec{u}}{2 c_{l k}^{2}}\right)\right],
\end{gathered}
$$

One of the lattice method's strengths is dealing with complicated boundary conditions. It is easy to apply a complicated or moving solid wall boundary condition, such as phase interface, to lattice methods for flow simulation. Halfway bounce back treatment is a widely used simple boundary condition for lattice methods [49-51], which requires the solid boundary to be in the middle of two lattices and offers second-order accuracy. For practical problems, especially a curved boundary, the solid boundary cannot always be located at the middle of two lattices perfectly. Hence, for a more accurate and smoother velocity field near a curved boundary without requiring high mesh resolution, Filipova and Hänel (FH) offer a curved wall boundary condition [52], which is further improved by Mei, Luo and Shyy (MLS). Bao, Yuan, and Schaefer further refine this technique to solve the mass conserving problem when body force is applied on the fluid field [53]. This boundary condition treatment is used in the proposed model. For a moving boundary, such as the phase interface, the momentum source from a moving wall can be incorporated into a distribution function as $2 w_{i l} \rho \frac{3}{c_{l k}^{2}} \vec{e}_{i l} \vec{u}_{\text {wall }}$. This implicit lattice kinetics method has the advantage of being stable at larger time steps but requires an iterative 
solution. The detailed iteration method and steps are introduced in Rector's work [26].

An implicit finite difference method with uniform mesh size is used to solve the system of governing equations for energy and phase field. Jacobi interaction method is used to solve the implicit finite difference system, so for updating the variables on each grid, only 6 nearby grids are needed for three-dimensional case. For parallel computation, the whole domain is equally divided by the total number of computing cores into blocks. Therefore, only one layer of data near the interface between blocks is needed to be transmitted between computing cores. The main steps for crystal growth simulation are: (1) calculate the velocity profile in a lattice kinetics scheme with the known temperature from the last time step to estimate the buoyancy force; (2) calculate the temperature profile with the known velocity profile from Step 1; (3) calculate the phase field with the known temperature profile from Step 2; (4) update the position of the phase interface and construct the liquid and solid domain; and (5) go to next time step. We use the ParaFlow program, a large-scale parallel CFD program based on the semi-implicit lattice method [32], to solve the fluid flow and temperature field and combine it with the parallel finite difference solver as briefly introduced above for solving the phase field. Because the coupled system is solved explicitly, the time step interval $\Delta t$ is on the order of $10^{-2}$ to $10^{-3}$ seconds for the numerical scheme stability. A two-dimensional benchmark test was demonstrated in our previous work [22], and the simulation results can match the analytical solution very well.

\section{Results and Discussion}

For a quantitative examination of the quality of a crystal, the variance of a chemical component's molar fraction is defined as:

$$
\operatorname{var}(c)=\frac{1}{\Omega_{g}} \int_{\Omega_{g}}[c-\operatorname{avg}(c)]^{2} d \Omega_{g}
$$

where $\Omega_{g}$ is the volume of grown crystal and $\operatorname{avg}(c)$ is the average molar fraction in grown crystal, which is defined as:

$$
\operatorname{avg}(c)=\frac{1}{\Omega_{g}} \int_{\Omega_{g}} c d \Omega_{g}
$$

Generally, a smaller variance of the molar fraction in grown crystal means more uniform distribution in crystal, and it indicates a higher quality of crystal. 


\subsection{Simulations of crystal growth in vertical gradient furnaces with ACRT}

This section presents the results from numerical simulations of crystal growth in vertical gradient furnaces. Figure 1 depicts the cylindrical simulation domain, in which the radius $(R)$ at the crucible is $0.76[\mathrm{~cm}]$, and the height $(H)$ is $9.6[\mathrm{~cm}]$. An $80 \times 80 \times 480$ D3Q19 lattice mesh is generated for containing the cylindrical crucible, so the total number of active lattices is about 2.4 million. The lattice size is $2 \times 10^{-4}[\mathrm{~m}]$, and interface thickness $\left(W_{0}\right)$ is $423 d_{0}$. The heat transfer coefficient, $h=150.0\left[\mathrm{~W} \mathrm{~m}^{-2} \mathrm{~K}^{-1}\right]$, is used in the boundary condition as shown in Equation (13). The solidification of the melt in the furnace is controlled by the temperature profile $T_{w}(z)$, which is shown in Equation(14). For hot-end temperature, $T_{h}=1223[K]$, and, for cold-end temperature, $T_{c}=1154[K]$. For initial conditions, $\vec{u}=0, \psi=-1$, the mole fraction concentration field $c=0.1$ in liquid, $\psi=1, c=0.135$ in solid, and the initial temperature is calculated from Equation (14). The Reynolds number of the system is about $2 \times 10^{4}$. As such, the $k-\epsilon$ turbulence model is incorporated with the proposed semi-implicit lattice kinetics model via Filippova's approach [54]. At the beginning, a flat solid-liquid interface is set at $z_{i 0}=2[\mathrm{~cm}]$, below which is solid phase, and above which is liquid phase. The initial temperature in the ampoule is assumed to be the same as boundary calculated by Equation (14). As the ampoule rotates along the center axis with the rotation cycle as demonstrated in Figure 3, the rotation rate gradually changes between -10 and 10 rounds per minute. Tables 1 and 2 provide the physical properties and non-dimensionless numbers used in the simulations.

Figure 5 shows the streamlines in the three-dimensional domain after 0,144 , and 288 seconds growth with the pulling speed $U_{\text {pull }}=1.3336^{-5}\left[\mathrm{~m} \mathrm{~s}^{-1}\right]$. The streamline colors represent the magnitude of velocity $U=\sqrt{u^{2}+v^{2}+w^{2}}$, where $u, v$, and $w$ are velocity components in the $x, y$, and $z$ directions, respectively. Figure 6 shows the streamlines on the center slice after 0, 72, 144, 216, and 288 seconds. The velocity magnitude shown in Figure 6 is calculated using $U=\sqrt{u^{2}+w^{2}}$, which eliminates the velocity perpendicular to the displayed slice and illustrates vertical transport in the liquid phase more clearly. It is obvious that the weak circulation near the solid-liquid interface disappears when the ampoule rotates at high speed, such as at 72 and 216 seconds. Figure 7 shows the zinc molar fraction distribution during crystal growth. The variance of the zinc molar fraction in the grown crystal with ACRT rotation is $1.35 \times 10^{-5}$ after 288 seconds of growth. The variance for the case without ACRT rotation is $1.39 \times 10^{-5}$. Hence, ACRT can slightly decrease the zinc molar fraction distribution variance in grown crystal, which means ACRT can be a benefit to the quality of crystal growth for the vertical gradient furnace. 


\subsection{Simulations of crystal growth in vertical gradient furnaces with TMF}

The stationary magnetic field and Lorentz force field are calculated from Equations (8)-(11) by finite difference scheme in an axisymmetric domain, as shown in Figure 2. There are 8 coils encircling the ampoule, and the distance between the coils is $1.24[\mathrm{~cm}]$. Table 1 provides the physical properties for magnetic field simulation. Figure 2 shows the in-phase, and out-of-phase, as well as the Lorentz force field, which are solved in finite difference scheme. The axisymmetric domain of the Lorentz force field result is extruded to a Cartesian threedimensional coordinate system then applied to the crystal growth simulation, as introduced in Equation (35). The simulation of crystal growth is based on the same geometric configuration as introduced in Section 4.1. Figure 8(a) compares the streamlines for cases with and without TMF. The Lorentz force obviously decreases the strength of the large and strong circulation, but it cannot affect the weak circulation near the solid-liquid interface. Figure 8(b) shows the zinc molar fraction. Although the TMF can affect concentration distribution in large areas of the liquid phase, it cannot significantly affect molar fraction in the area near the solid-liquid interface and the crystal. The variance of the molar fraction in grown crystal with TMF after 288 seconds is $1.41 \times 10^{-5}$, which is greater than the case without TMF $\left(1.39 \times 10^{-5}\right)$. The Lorentz force field direction is opposite to the strong circulation, but it has the same direction as the weak circulation near the solid-liquid interface. Hence, Lorentz force slightly accelerates weak circulation, decreasing the quality of the grown crystal. Yesilyurt's work [36] shows that the TMF can decrease velocity shear near the solid-liquid interface for the linear temperature gradient furnace. A furnace with a temperature gradient like the one introduced in Equation (14) cannot benefit from TMF. In examining the effect of TMF, due to CZT's low electrical conductivity, a very large electric current density $\left(J_{0}=7.5 \times 10^{7}\left[\mathrm{~A} \mathrm{~m}^{-2}\right]\right)$ is used, which may be difficult to achieve under common experimental conditions. For other materials, such as germanium $(\mathrm{Ge})$ and gallium arsenide (GaAs), a more realistic electric current density can make the Lorentz force field strong enough to counter the buoyancy force.

\subsection{Efficiency of the parallel computation}

As mentioned in the previous section, a primary advantage of lattice-based CFD methods is their inherent parallelism [26,32]. High Rayleigh number convection in the melt region requires a small time step. Also, crystal growth is a very slow procedure, often lasting several hours to a few days. Crystal growth simulation is computationally expensive when the Rayleigh number is high, especially for a three-dimensional simulation. The simulation program was tested on the PNNL Institutional Computing (pic.pnnl.gov), which includes 
2400 AMD Interlagos 16-core processors. Figure 9 depicts the speedup for the program running on 80,160, 320, and 640 central processing unit (CPU) cores. The program shows considerable speedup and great acceleration potential as the number of CPU cores increases.

\section{Summary and Discussion}

A coupled lattice kinetics phase-field model for crystal growth in a vertical gradient furnace has been developed. The model accounts for buoyancy, ACRT, and TMF-induced convective flow and their effects on the crystal growth process. This makes the phase-field model applicable for a wide range of freeboundary problems, including modeling the macroscale interface evolution during solidification in the vertical gradient furnace. A one-way coupled concentration transport model is used for solving the molar fraction of the chemical component in both the liquid and solid phases, and the boundary condition at the solid-liquid interface described in Equation (17) can be applied automatically instead of tracking the position of the interface. Therefore, using a continuous phase-field to simulate a chemical concentration field that has a jump cross the interface provides an efficient way to examine the quality of the crystal growth. The chemical component's concentration in the solid phase can be used to access the quality of crystal growth for different practical applications, such as applying ACRT and TMF to the solidification system. A semi-implicit lattice kinetics method is used for solving convection in the melt region. Through demonstration cases, it is shown that ACRT can slightly increase the quality of grown crystal. However, the effect of TMF on quality of grown crystal depends on the temperature profile of the ampoule wall. A furnace with a temperature gradient like the one introduced in Equation (14) cannot benefit from TMF. Finally, excellent scalability of our developed parallel methods is demonstrated on the three-dimensional cases.

\section{Acknowledgments}

This work was supported by the Applied Mathematics Program within the Department of Energy Office of Advanced Scientific Computing Research as part of the Collaboratory on Mathematics for Mesoscopic Modeling of Materials. PNNL is operated by Battelle for the U.S. Department of Energy under Contract DE-AC05-76RL01830. We would like to thank Dr. Kenneth Jarman for carefully proofreading the manuscript. The research was performed using PNNL Institutional Computing, as well as the National Energy Research Scientific Computing Center at Lawrence Berkeley National Laboratory. 
Table 1

Physical properties of the growing crystal and melting solution used in the simulations of crystal growth in the vertical gradient furnace.

\begin{tabular}{|l|c|}
\hline Physical parameter & Values \\
\hline Specific heat $C_{p}\left[\mathrm{~J} \mathrm{~kg}^{-1} \mathrm{~K}^{-1}\right]$ & 380 \\
Thermal conductivity $K\left[\mathrm{~W} \mathrm{~m}^{-1} \mathrm{~K}^{-1}\right]$ & 42.8 \\
Mass density $\rho\left[\mathrm{kg} \mathrm{m}^{-3}\right]$ & 5633 \\
Thermal expansion $\beta_{T}\left[\mathrm{~K}^{-1}\right]$ & $1.2 \times 10^{-4}$ \\
Viscosity $\mu\left[\mathrm{kg} \mathrm{m}^{-1} \mathrm{~s}^{-1}\right]$ & $7.35 \times 10^{-4}$ \\
Melting temperature $[\mathrm{K}]$ & 1211.4 \\
Latent heat $L\left[\mathrm{~J} \mathrm{kg^{-1 } ]}\right.$ & $4.65 \times 10^{5}$ \\
Surface tension $\vartheta\left[\mathrm{kg} \mathrm{s}^{-2}\right]$ & 0.554 \\
Diffusion coefficient in liquid phase $D_{l}\left[\mathrm{~m}^{2} \mathrm{~s}^{-1}\right]$ & $1.0 \times 10^{-8}$ \\
Diffusion coefficient in solid phase $D_{s}\left[\mathrm{~m}^{2} \mathrm{~s}^{-1}\right]$ & $1.0 \times 10^{-8}$ \\
Segregation coefficient $k$ & 1.35 \\
Magnetic permeability $\mu_{m}\left[H \mathrm{~m}^{-1}\right]$ & $1.26 \times 10^{-6}$ \\
Electric conductivity $\sigma\left[S \mathrm{~m}^{-1}\right]$ & $1.0 \times 10^{4}$ \\
Angular frequency $\omega[\mathrm{Hz}]$ & $2 \pi \times 50$ \\
Phase shift $\phi_{n}$ & $\pi / 4$ \\
\hline
\end{tabular}

Table 2

Non-dimensionless numbers and their characteristic values used in the simulations of crystal growth in the vertical gradient furnace.

\begin{tabular}{|l|c|c|}
\hline Dimensionless numbers & Expression & Characteristic values \\
\hline Rayleigh number & $\operatorname{Ra}=\frac{g \beta_{T} \Delta T L^{3}}{\mu \alpha_{L}}$ & $1.81 \times 10^{5}$ \\
Prandtl number & $\operatorname{Pr}=\mu / \alpha$ & $6.53 \times 10^{-3}$ \\
\hline
\end{tabular}




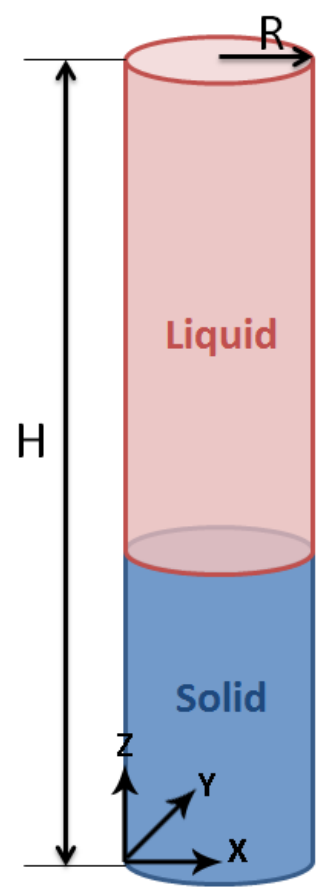

Fig. 1. Schematic of computational domain.
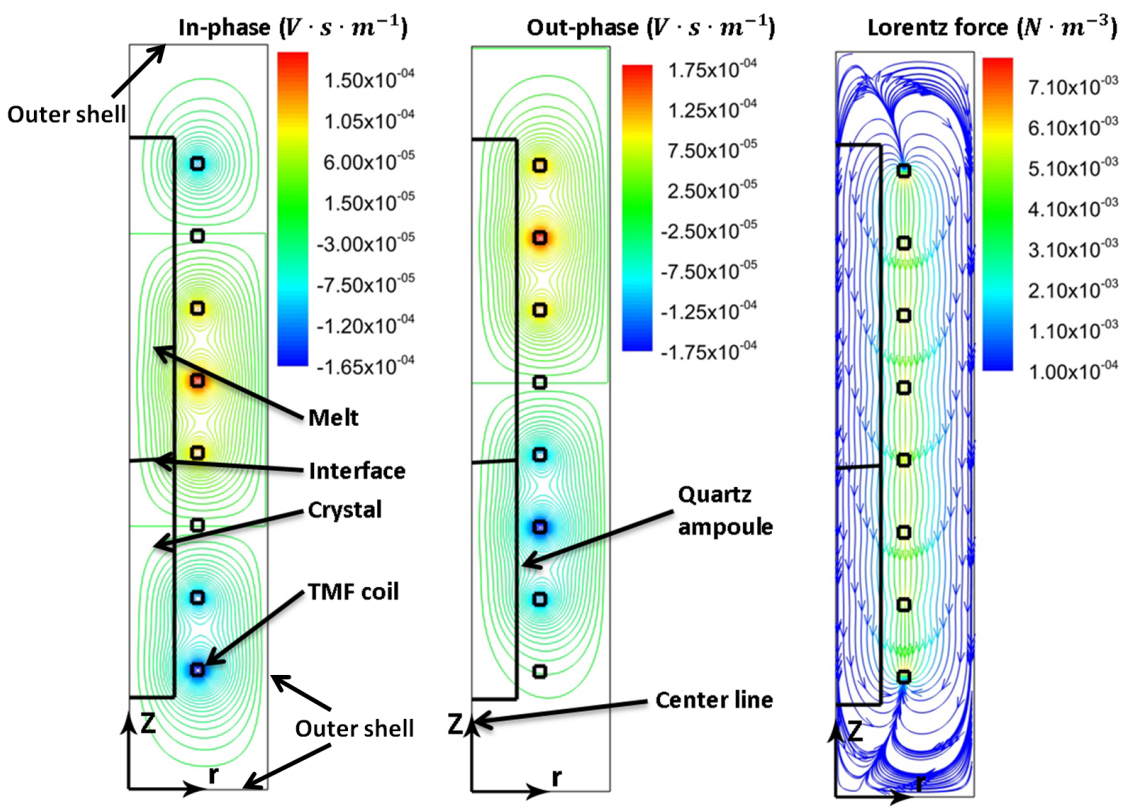

Fig. 2. Demonstration of in-phase and out-of-phase components and Lorentz force applied on the furnace. 


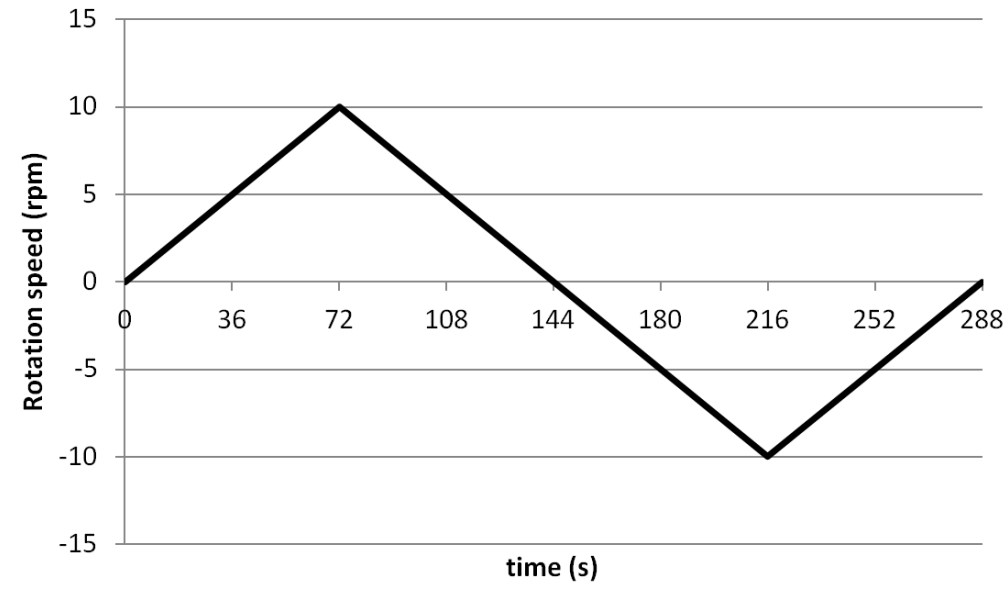

Fig. 3. ACRT rotation cycle.

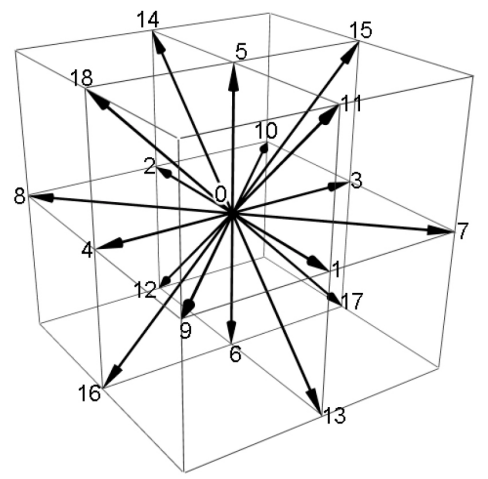

Fig. 4. D3Q19 lattice. 


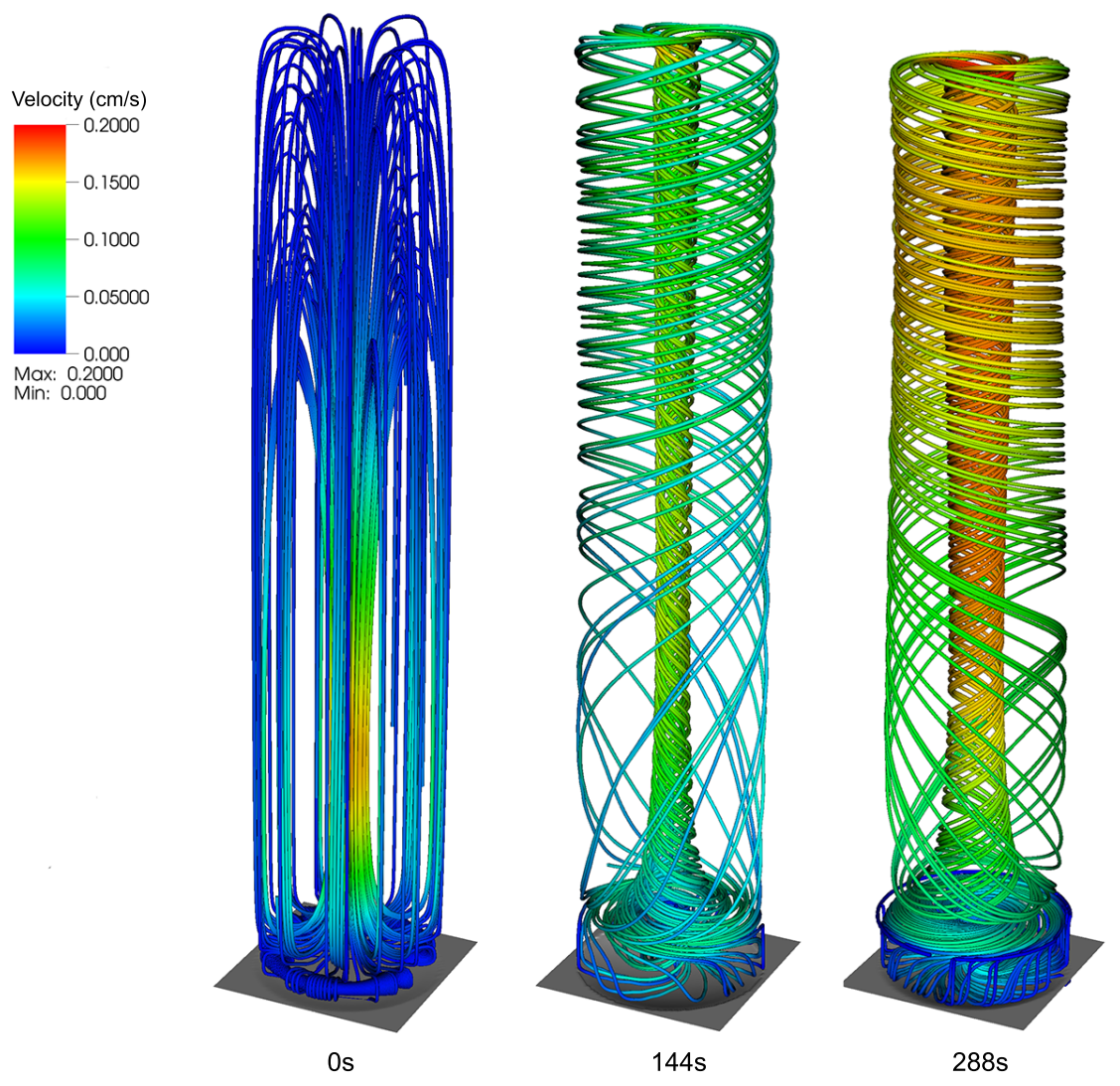

Fig. 5. Streamlines in the liquid phase in an ACRT rotation cycle. 


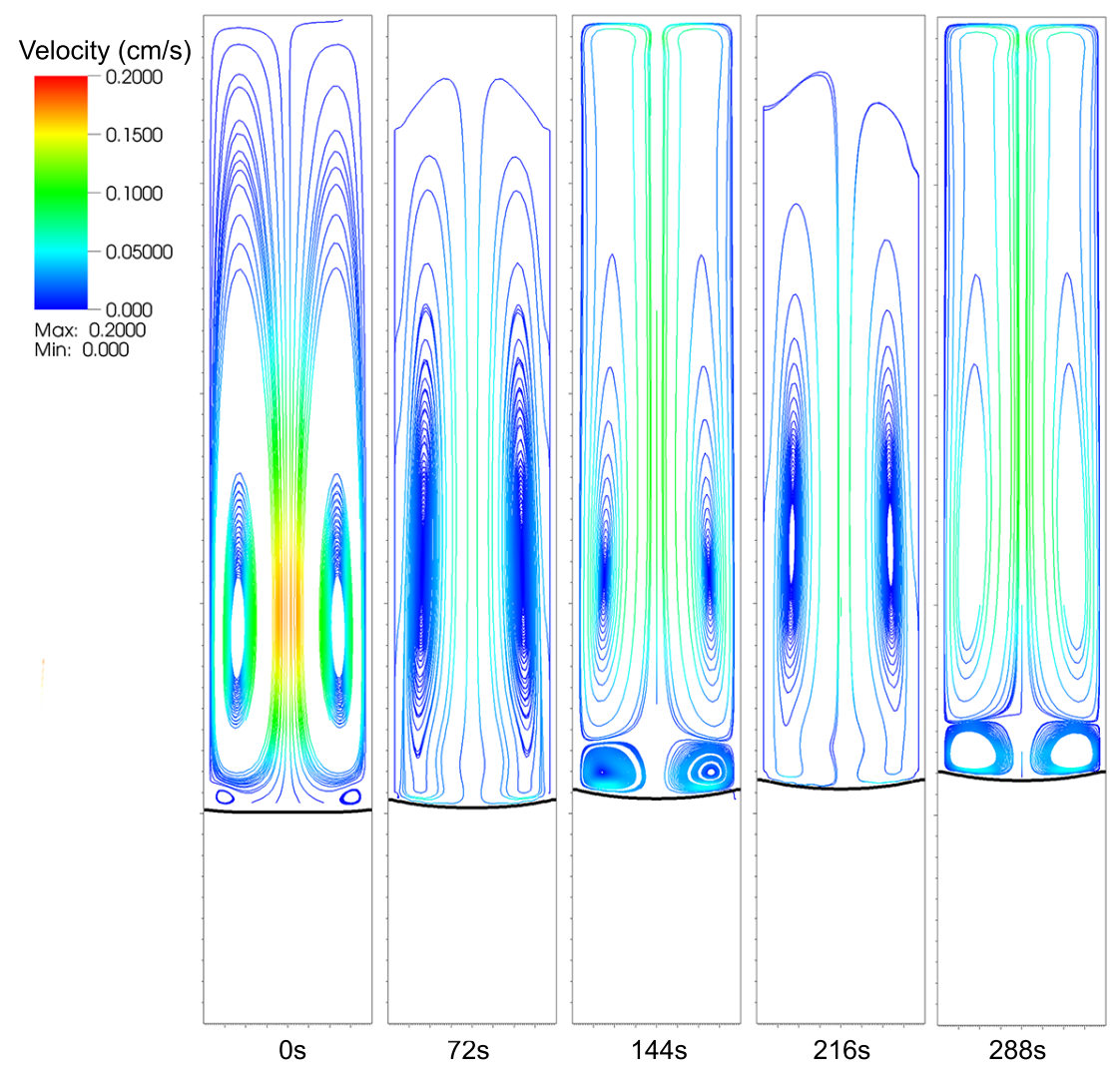

Fig. 6. Streamlines at the center slice at 0, 72, 144, 216, and 288 seconds with ACRT rotation. 


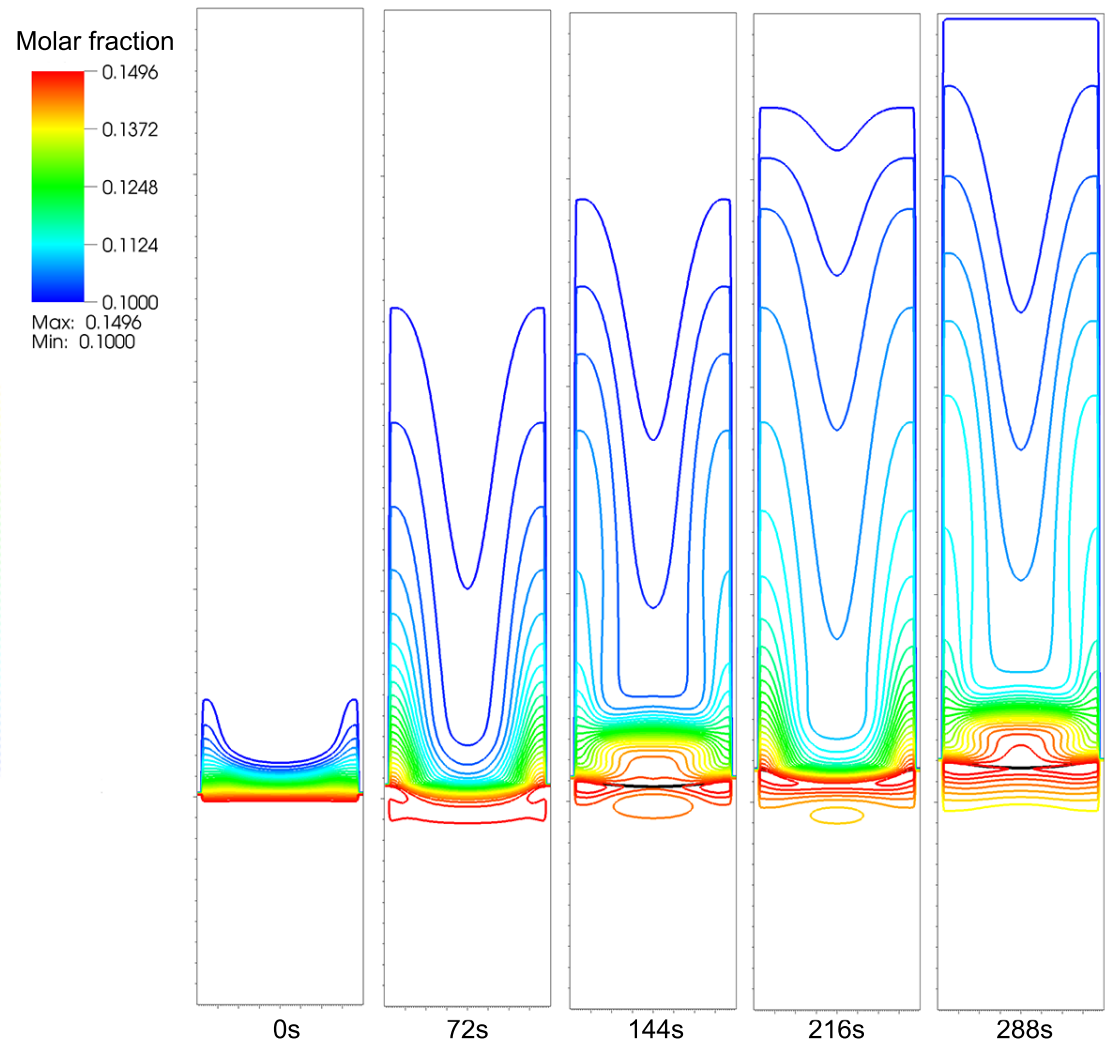

Fig. 7. Zinc molar fraction at $0,72,144,216$, and 288 seconds with ACRT rotation. 
(a)
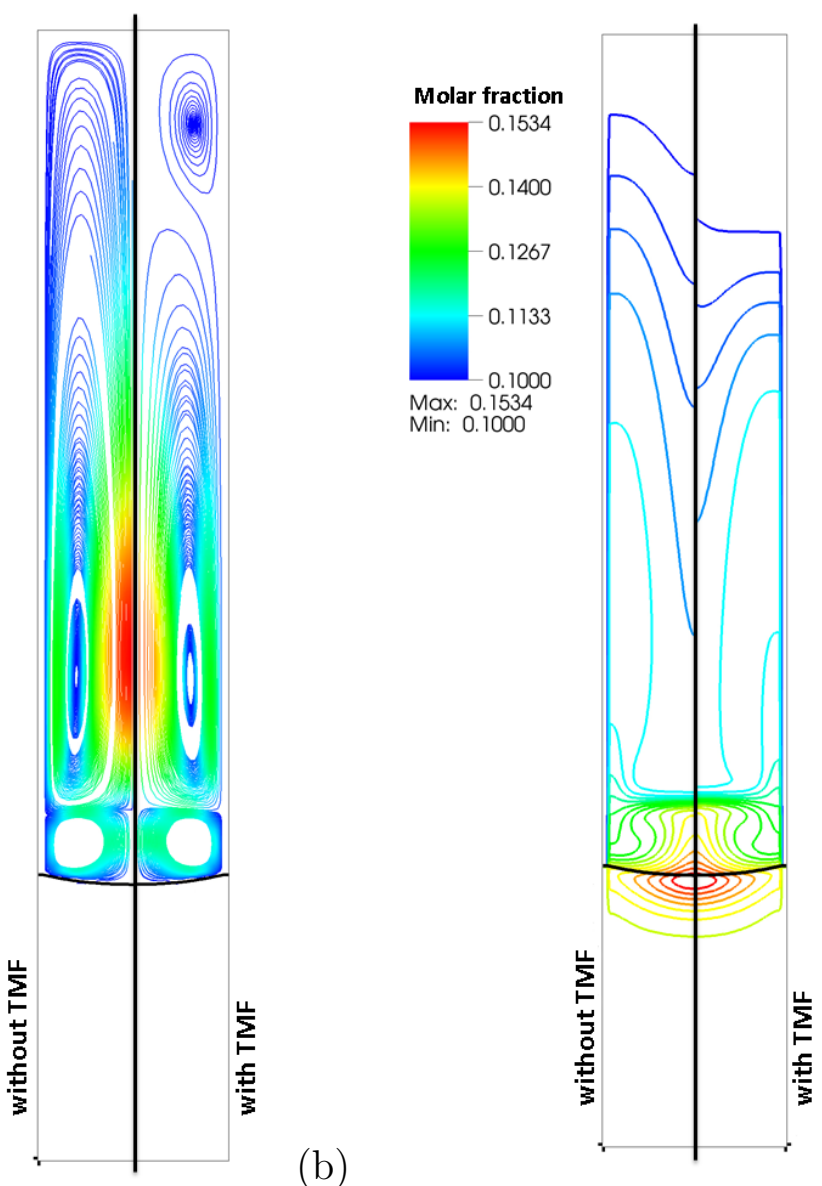

(b)

Fig. 8. (a) Streamlines for the furnace with and without TMF after 288 seconds of growth. (b) Zinc molar fraction for the furnace with and without TMF after 288 seconds of growth.

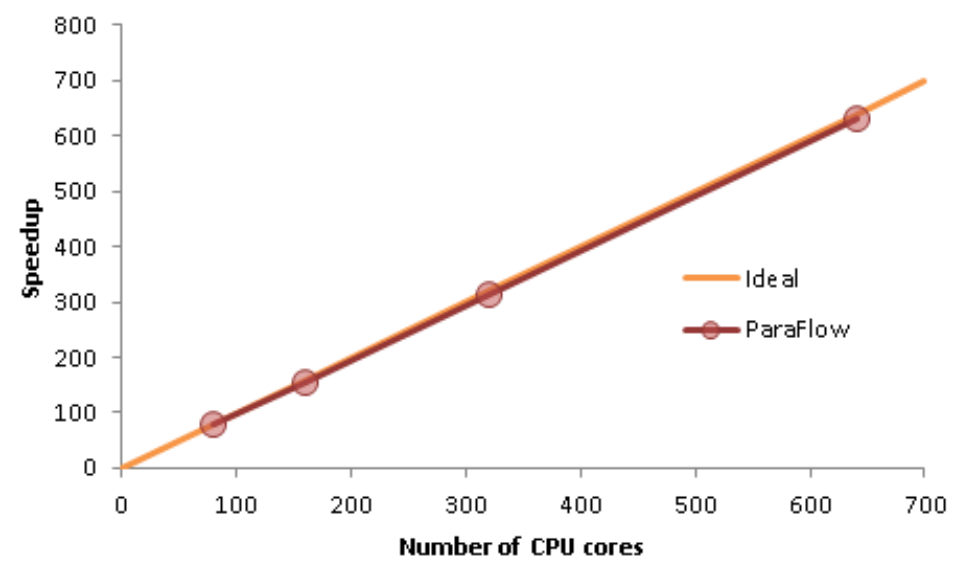

Fig. 9. Speedup for the ParaFlow program running on 80, 160, 320, and $640 \mathrm{CPU}$ cores. 


\section{References}

[1] U. Becker, H. Zimmermann, P. Rudolph, R. Boyn, Optical study of the impurity distribution in vertical-Bridgman-grown CdTe crystals, Phys. Status Solidi (A) Applied Research 112 (2) (1989) 569-578.

[2] H. Hermon, M. Schieber, R. James, J. Lund, A. Antolak, D. Morse, N. Kolesnikov, Y. Ivanov, M. Goorsky, H. Yoon, J. Toney, T. Schlesinger, Homogeneity of CdZnTe detectors, Nuclear Instruments \& Methods in Physics Research, Section A: Accelerators, Spectrometers, Detectors and Associated Equipment 458 (1) (2001) 100-106.

[3] H. Hermon, M. Schieber, E. Lee, J. McChesney, M. Goorsky, T. Lam, E. Meerson, H. Yao, J. Erickson, R. James, CZT detectors fabricated from horizontal and vertical Bridgman-grown crystals, Nuclear Instruments \& Methods in Physics Research, Section A: Accelerators, Spectrometers, Detectors and Associated Equipment 410 (1) (1998) 503-510.

[4] M. Yildiz, S. Dost, B. Lent, Growth of bulk SiGe single crystals by liquid phase diffusion, J. Cryst. Growth 280 (1) (2005) 151160.

[5] M. Yildiz, S. Dost, A continuum model for the liquid phase diffusion growth of bulk SiGe single crystals, International Journal of Engineering Science 43 (2005) 1059-1080.

[6] P. Sonda, A. Yeckel, P. Daoutidis, J. J. Derby, Development of model-based control for Bridgman crystal growth, Journal of Crystal Growth 266 (1) (2004) $182-189$.

[7] J. Derby, L. Lun, A. Yeckel, Strategies for the coupling of global and local crystal growth models, Journal of Crystal Growth 303 (1) (2004) 114-123.

[8] L. Lun, A. Yeckel, P. Daoutidis, J. Derby, Decreasing lateral segregation in cadmium zinc telluride via ampoule tilting during vertical Bridgman growth, Journal of Crystal Growth 291 (2) (2006) 348-357.

[9] P. Sonda, A. Yeckel, J. Derby, P. Daoutidis, The feedback control of the vertical Bridgman crystal growth process by crucible rotation: two case studies, Computers and Chemical Engineering 29 (2005) 887-896.

[10] P. Sonda, A. Yeckel, P. Daoutidis, J. Derby, Hopf bifurcation and solution multiplicity in a model for destabilized Bridgman crystal growth, Chem. Eng. Sci. 60 (2005) 1323-1336.

[11] A. Pandy, A. Yeckel, M. Reed, C. Szeles, M. Hainke, G. Mller, J. Derby, Analysis of the growth of cadmium zinc telluride in an electrodynamic gradient freeze furnace via a self-consistent, multi-scale numerical model, Computers and Chemical Engineering 276 (2005) 133-147.

[12] T. Nishinaga, Microchannel epitaxy: an overview, J. Cryst. Growth 237 (2002) 1410-1417. 
[13] Y. Liu, Z. Zytkiewicz, S. Dost, A model for epitaxial lateral overgrowth of GaAs by liquid phase electroepitaxy, J. Cryst. Growth 265 (4) (2004) 341-350.

[14] K. Mazuruk, N. Ramachandran, M. Volz, Proceedings of the 37th AIAA Aerospace Sciences Meeting Reno, Nevada AIAA 99-0941.

[15] R. Grugel, K. Mazuruk, Proceedings of the 39th AIAA Aerospace Sciences Meeting Reno, Nevada AIAA 01-0462.

[16] A. Yeckel, J. Derby, Effects of a traveling magnetic field on vertical gradient freeze growth of cadmium zinc telluride, proceedings of hard X-ray, Gamma-ray, and neutron detector physics XIII, SPIE optical engineering + applications.

[17] A. Yeckel, J. Derby, Effect of accelerated crucible rotation on melt composition in high-pressure vertical Bridgman growth of cadmium zinc telluride, J. Cryst. Growth 209 (1999) 737-750.

[18] P. Capper, J. Gosney, C. Jones, Application of the accelerated crucible rotation technique to the Bridgman growth of CdxHg1-xTe: Simulations and crystal growth, J. Cryst. Growth 70 (1984) 356-364.

[19] W. Coates, P. Capper, C. Jones, J. Gosney, C. Ard, I. Kenworthy, A. Clark, Effect of ACRT rotation parameters on Bridgman grown CdxHg1-xTe crystals, J. Cryst. Growth 94 (1989) 959-966.

[20] P. Capper, J. Gosney, C. Jones, I. Kenworthy, Bridgman growth of Cdx Hg1-xTe using ACRT, J. Electron. Mater. 15 (1986) 371-376.

[21] C. Lan, J. Chian, Effects of ampoule rotation on vertical zone-melting crystal growth: steady rotation versus accelerated crucible rotation technique (ACRT), J. Cryst. Growth 203 (1999) 286-296.

[22] G. Lin, J. Bao, Z. Xu, A. M. Tartakovsky, C. H. J. Henager, A phase-field model coupled with lattice kinetics solver for modeling crystal growth in furnaces, Communications in Computational Physics In press.

[23] G. Fix, Free Boundary Problems: Theory and Applications, Piman, Boston, 1983.

[24] A. Karma, W.-J. Rappel, Quantitative phase-field modeling of dendritic growth in two and three dimensions, Physical review E 57 (4) (1998) 4323-4349.

[25] B. Echebarria, R. Folch, A. Karma, M. Plapp, Quantitative phase-field model of alloy solidification, Physical Review E 70 (2004) 0616041-06160422.

[26] D. Rector, M. Stewart, A semi-implicit lattice method for simulating flow, J. Comp. Phys. 229 (2010) 6732-6743.

[27] R. Benzi, S. Succi, M. Vergassola, The lattice Boltzmann equation: theory and applications, Phys. Rep. 222 (1992) 145.

[28] S. Chen, G. Doolen, Lattice Boltzmann method for fluid flows, Ann. Rev. Fluid Mech. 30 (1998) 329-364. 
[29] D. Yu, R. Mei, L. Luo, W. Shyy, Viscous flow commutations with the method of lattice Boltzmann equation, Progr. Aerosp. Sci 39 (2003) 329-367.

[30] S. Succi, The lattice Boltzmann equation for fluid dynamics and beyond, Oxford University Press, Oxford, 2001.

[31] T. Inamuro, A lattice kinetic scheme for incompressible viscous flows with heat transfer, Philos. T. Roy. Soc. A 360 (2002) 477-484.

[32] D. Rector, M. Stewart, J. Bao, Modeling of HLW tank solid resuspension and mixing process, WM2102 Conference, Phoenix, AZ, 2012.

[33] L. Arkeryd, On the Boltzmann equation part I: Existence, Arch. Rational Mech. Anal. 45 (1972) 1-16.

[34] L. Arkeryd, On the Boltzmann equation part II: The full initial value problem, Arch. Rational Mech. Anal. 45 (1972) 1734.

[35] R. DiPerna, P. Lions, On the Cauchy problem for Boltzmann equations: global existence and weak stability, Ann. Of Math. 130 (2) (1989) 321366.

[36] S. Yesilyurt, S. Motakef, R.Grugel, K. Mazuruk, The effect of the traveling magnetic field (TMF) on the bouyancy-induced convection in the vertical Brgrowth of semiconductors, J. Crystal Growth 263 (2004) 80-89.

[37] H. Lee, A. Pearlstein, Simulation of radial dopant segregation in vertical Bridgman growth of GaSe, a semiconductor with anisotropic solid-phase thermal conductivity, J. Crystal Growth 231 (2001) 148-170.

[38] H. Lee, A. Pearlstein, Simulation of vertical Bridgman growth of benzene, a material with anisotropic solid-phase thermal conductivity, J. Crystal Growth 209 (2000) 934-952.

[39] S. Unverdi, G. Tryggvason, A front-tracking method for viscous, incompressible multi-fluid flows, J. Comput. Phys. 100 (1992) 25-37.

[40] F. D. Sousa, N. Mangiavacchi, L. Nonato, A. Castelo, M. Tom, V. Ferreira, S. M. J.A. Cuminato, A front-tracking/front-capturing method for the simulation of 3D multi-fluid flows with free surfaces, J. Comput. Phys. 198 (2004) 469-499.

[41] S. Popinet, S. Zalesk, A front tracking algorithm for accurate representation of surface tension, Int. J. Numer. Meth. Fluid 30 (1999) 775793.

[42] X. He, L. Luo, A priori derivation of the lattice Boltzmann equation, Phys. Rev. E 55 (1997) R6333-R6336.

[43] Z. Guo, C. Zheng, B. Shi, Discrete lattice effects on the forcing term in the lattice Boltzmann method, Phys. Rev. E 65 (2002) 046308-046314.

[44] D. Wolf-Gladrow, Lattice-gas cellular automata and lattice Boltzmann models: an introduction, Springer-Verlag, Germany.

[45] X. He, L. Luo, Lattice Boltzmann model for the incompressible Navier-Stokes equation, J. Stat. Phys. 88 (1997) 927-944. 
[46] A. Karma, Phase-Field formulation for quantitative modeling of alloy solidification, Phys. Rev. Lett. 87 (2001) 115701-115705.

[47] Z. Xu, P. Meakin, Phase-field modeling of solute precipitation and dissolution, J. Chem. Phys. 129 (2008) 014705.

[48] Z. Xu, P. Meakin, Phase-field modeling of two-dimensional solute precipitation: radial solid fingers and diffusion-limited aggregation, J. Chem. Phys. 134 (2011) 044137.

[49] C. Aidun, Y. Lu, Lattice Boltzmann simualtions of solid particles suspended in fluid, J. Stat. Phys. 81 (1995) 49-61.

[50] A. Ladd, Numerical simualtions of particulate suspensions via a discretized Boltzmann equation, J. Fluid Mech. 271 (1994) 285-339.

[51] D. Ziegler, Boundary Conditions for Lattice Boltzmann Simulations, J. Stat. Phys. 71 (1993) 1171-1177.

[52] O. Filippova, D. Hanel, Grid refinement for lattice-BGK models, J. Comp. Phys. 147 (1998) 219-228.

[53] J. Bao, P. Yuan, L. Schaefer, A mass conserving boundary condition for the lattice Boltzmann equation method, J. Comp. Phys. 227 (2008) 8472-8487.

[54] O. Filippova, S. Succi, F. Mazzocco, C. Arrighetti, G. Bella, D. Hanel, Multiscale Lattice Boltzmann Schemes with Turbulence Modeling, J. Comput. Phys. 170 (2001) 812-829. 